\title{
Fluoroscopia y protección radiológica en tratamiento del dolor
}

\author{
J. M. Hernández García ${ }^{1}$, M. A. Reina ${ }^{1}$ y A. Vidal $\operatorname{Marcos}^{2}$ \\ ${ }^{1}$ Departamento de Anestesiología y Tratamiento del Dolor. Hospital Universitario Madrid-Montepríncipe. \\ Madrid. ${ }^{2}$ Departamento de Anestesiología y Tratamiento del Dolor. Hospital Sur. Alcorcón, Madrid
}

Hernández García JM, Reina MA, Vidal Marcos A. Fluoroscopia y protección radiológica en tratamiento del dolor. Rev Soc Esp Dolor 2015; 22(5): 217-223.

\begin{abstract}
The use of fluoroscopy is an essential tool for the practice of blocks and techniques in pain management. However and despite its frequent use, a large number of pain specialists have not received specific training, according to the study that we carried out among pain practitioners (1).

In the present study we pretend to describe the basic principles of radiation, generation of $\mathrm{X}$ rays, and control of the monitor image using the, voltage or current. We also describe the dose limits established by Spanish law, how to monitor radiation, recommendations to reduce exposure to $\mathrm{X}$ rays and finally we insist on the importance to inform the patient about the use of $\mathrm{X}$ rays, since rarely it is done.
\end{abstract}

Key words: Fluoroscopy. Radiation safety.

\section{RESUMEN}

La fluorosocopia es una técnica esencial para la realización de la mayoría de bloqueos en tratamiento del dolor pero, a pesar de utilizarse diariamente, un gran porcentaje de especialistas no ha recibido formación específica, según se describe en una encuesta realizada entre los encuestados de la especialidad (1).

Con este artículo pretendemos exponer conceptos básicos de los tipos de radiación, sobre cómo se generan los rayos $\mathrm{X}$ y

Recibido: 01-12-14.

Aceptado: 15-03-15. cómo podemos controlar la imagen que obtenemos en el monitor modificando diferentes parámetros como corriente o voltaje. También se describen los límites de dosis establecidos en la legislación española, cómo se puede monitorizar la radiación, las recomendaciones para reducir la exposición a rayos X y tratamos de insistir en la importancia de informar al paciente del uso de rayos $\mathrm{X}$, lo cual raramente realizamos.

Palabras clave: Fluoroscopia. Protección radiológica.

\section{INTRODUCCIÓN}

La utilización de la fluoroscopia en tratamiento del dolor se ha establecido como técnica de buena praxis a la hora de realizar la mayoría de técnicas, bloqueos o inyecciones de nuestra práctica diaria.

Esto requiere unos conocimientos que en muchos casos no se enseñan como parte de la formación y que el especialista va adquiriendo a lo largo de los años, en muchos casos después de haber estado protegido de forma inadecuada. Esto lo hemos podido comprobar en el estudio que realizamos entre los profesionales que nos dedicamos al tratamiento del dolor y que demuestra la falta de formación y las pocas medidas de protección utilizadas (1).

Por otro lado, no suele ser parte de los temas o comunicaciones que se presentan en los Congresos o Reuniones de la especialidad que se desarrollan.

Con este trabajo pretendemos exponer los conceptos de básicos de la radiación ionizante, los métodos de protección radiológica y de medición de la radiación en la práctica diaria.

Al hacer una revisión del tema hemos podido comprobar que aparece como capítulo en algún libro de texto de tratamiento del dolor, aunque encontramos pocos artículos en la 
literatura de nuestra especialidad. Los que aparecen suelen estar relacionados con otras especialidades, como radiología intervencionista, cardiología o cirugía de columna.

\section{TIPOS DE RADIACIÓN}

Es necesario, para comenzar, conocer los tipos de radiación a los que estamos sometidos y que empleamos en nuestro trabajo.

La radiación se define como "el proceso de transmisión de ondas o partículas a través del espacio o de algún medio". En el caso de transmisión por ondas (electromagnéticas) la llamamos "radiación electromagnética". Tiene un espectro que, dependiendo de la longitud de onda, se clasifica en rayos gamma, rayos $\mathrm{X}$, luz ultravioleta visible, luz infrarroja, radar, microondas y ondas de radio (en orden creciente de longitud de onda) (Fig. 1).

La radiación electromagnética, al atravesar el material sobre el que actúa, es capaz de alterar los átomos, de forma que puede arrancar electrones, produciendo un ión (positivo o negativo). De esta manera se genera la radiación ionizante. Son radiaciones ionizantes los rayos X, las radiaciones alfa, beta y gamma.

Estas alteraciones pueden provocar reacciones y cambios químicos. Por ejemplo, son capaces de romper los enlaces químicos de las moléculas o generar cambios genéticos en células reproductoras.

Por otro lado existe la radiación no ionizante, que no es capaz de producir iones al interactuar con los átomos de un material. Es el caso de los campos electromagnéticos y las radiaciones ópticas, como son los rayos láser, los rayos infrarrojos, la luz visible y la radiación ultravioleta. Esta radiación no ionizante puede provocar calor y ciertos efectos fotoquímicos al actuar sobre el cuerpo humano.

Las personas estamos expuestas a las radiaciones ionizantes de forma natural, pues en algunos casos proceden de la naturaleza y en otros son generadas por el hombre.

La radiación que se encuentra en la naturaleza se llama "radiación de fondo" y puede proceder de diferentes fuentes, como la radiación cósmica, procedente del espacio exterior, o la radiación emitida por las sustancias radiactivas presentes en la corteza terrestre. Además, existe un tipo de radiación producida por isótopos radiactivos contenidos en el propio organismo humano, principalmente isótopos del carbono y del potasio. A ello hay que unir la radiación producida por el radón que inhalamos al respirar.

Dentro de las causas producidas por el hombre la principal fuente de irradiación son los procedimientos médicos (rayos X y medicina nuclear), cuya dosis sobre la población se podría equiparar a la emitida por la radiación cósmica. En el caso de las centrales nucleares, producen una dosis prácticamente nula sobre el público en general y una dosis muy pequeña y controlada sobre el personal de la central.

\section{¿CÓMO SE GENERAN LOS RAYOS X?}

Los rayos $\mathrm{X}$ son generados en un tubo de vidrio al vacío con dos electrodos en sus extremos. El cátodo (negativo) es un filamento de tungsteno por el que pasa una corriente (que se mide en $\mathrm{mA}$ ) y el ánodo (positivo) es una placa de cobre. Los electrones generados en el cátodo son enfocados y acelerados por medio de alto voltaje (pico kilovoltio o $\mathrm{kVp}$ ) hacia el ánodo (que por lo general posee una inclinación de $45^{\circ}$ ) y producto de la colisión se generan los rayos X (Fig. 2).

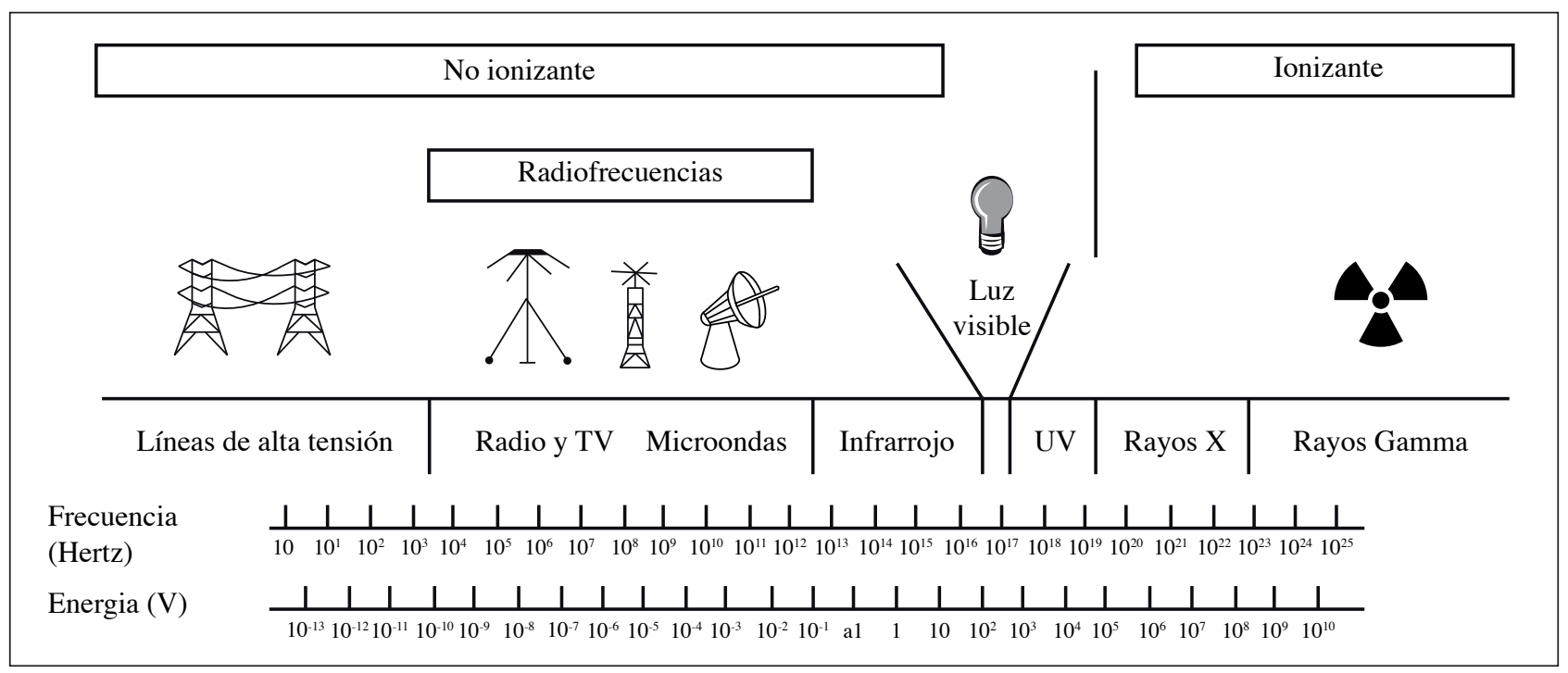

Fig. 1. Radiaciones electromagnéticas 


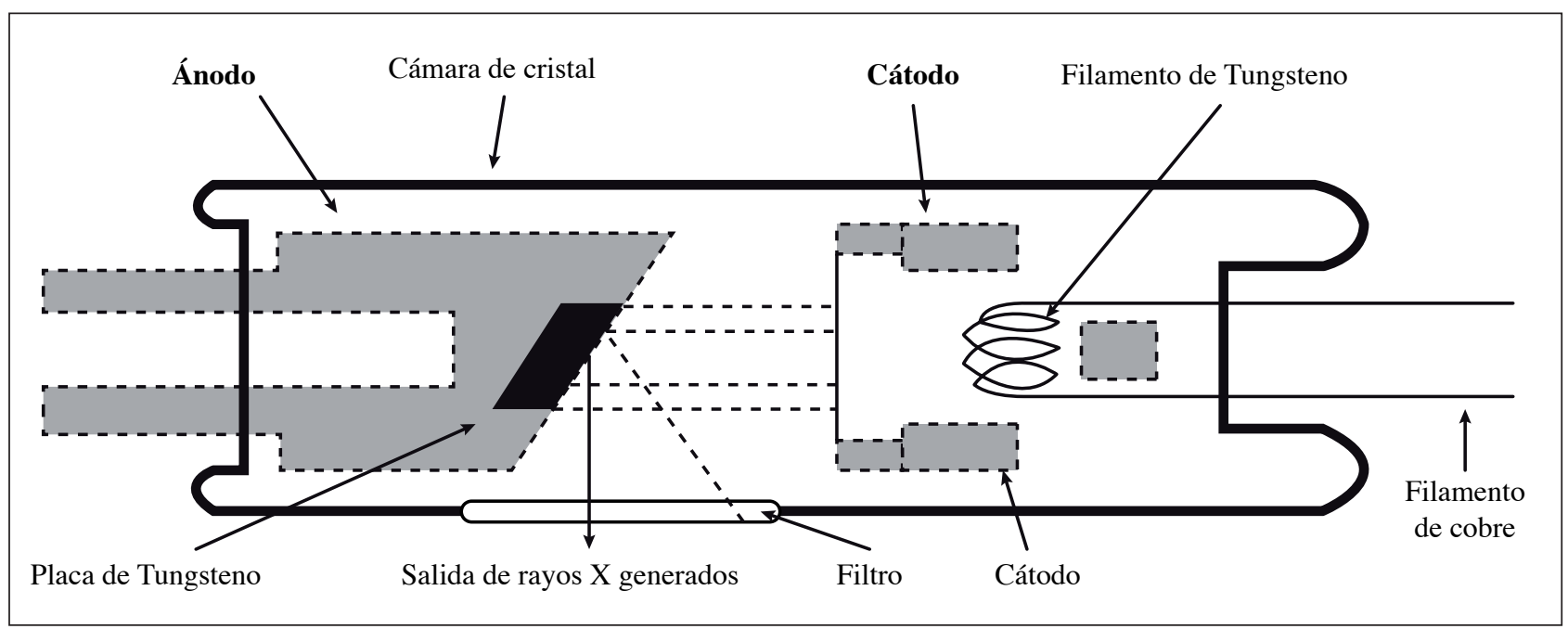

Fig. 2. Tubo de rayos X.

En fluoroscopia, los rayos $\mathrm{X}$ que entran y salen del paciente llegan al intensificador de imagen, que procesa la señal, y se convierten en una imagen que puede ser mostrada en un monitor.

En la radiografía los rayos $\mathrm{X}$ que pasan a través del cuerpo sin interaccionar son los que producen la imagen.

Hay varias maneras de controlar la salida de rayos $\mathrm{X}$ (Tabla I):

- Un aumento de la corriente (mA) provoca un aumento de electrones que salen hacia el ánodo y produce un mayor número de rayos $\mathrm{X}$.

- Junto con la corriente, el tiempo de exposición (segundos) afecta la cantidad de radiación que llega al intensificador de imagen. La densidad radiográfica, llamada a veces "densidad óptica”, es el grado de negro de la imagen radiográfica. En el caso de la placa, una imagen que aparece muy ennegrecida es porque está sobreexpuesta, debido a que ha llegado mucha radiación. En el caso de la fluoroscopia sería al revés, el intensificador de imagen convierte los rayos $\mathrm{X}$ que llegan en fotones de luz, de modo que una imagen sobreexpuesta en este caso aparece muy clara y viceversa.

TABLA I. FACTORES DE CONTROL DE LA IMAGEN DE RAYOS X

1. Corriente (mA): un aumento produce mayor cantidad de rayos $\mathrm{X}$

2. Tiempo de exposición: (seg) un aumento produce un ennegrecimiento de la imagen

3. Voltaje $(\mathrm{kVp})$ : un aumento produce aceleración de electrones y más rayos $\mathrm{X}$

4. Colimación: circular o rectangular
- Un aumento del voltaje $(\mathrm{kVp})$ produce un aumento de la aceleración de los electrones y mayor energía y radiación X (lo mismo que un aumento de la corriente, mA). En fluoroscopia, los valores que se utilizan normalmente son 2-6 mA y 75-125 kVp y en el caso de radiografía se utilizan normalmente corrientes de 50-1200 mA con tiempos de exposición muy cortos (2).

- La colimación limita el foco de rayos X a la zona anatómica requerida, al mismo tiempo que reduce la radiación dispersa y mejora la calidad de la imagen. Existen dos tipos de colimadores en fluoroscopios: unos producen un campo rectangular y otros, llamados "de abertura variable" (colimador tipo iris), produce un campo circular.

Existe además un filtro que la mayoría de equipos de rayos $\mathrm{X}$ tienen, formado por capas de aluminio y que se utiliza para eliminar los rayos $\mathrm{X}$ de baja energía que son absorbidos por el paciente pero no contribuyen a la formación de la imagen. Esto reduce la exposición del paciente a la radiación.

\section{CONTROL DE LA CALIDAD DE IMAGEN}

Para mejorar la imagen del monitor los equipos modernos de fluoroscopia utilizan el control automático de brillo, que ajusta de forma automática los $\mathrm{kVp}$ y $\mathrm{mA}$, obteniendo una imagen con el contraste, el brillo y la resolución óptima, aunque esto se puede ajustar también de forma manual.

Ajustando el voltaje $(\mathrm{kVp})$ se puede cambiar el contraste de la imagen para reducir la diferencia entre distintos tejidos (hueso y partes blandas): un aumento de $\mathrm{kVp}$ produce una imagen con mayor claridad en el monitor (disminuye el contraste y aumenta la penetración de los rayos X); lo 
contrario, disminuir el $\mathrm{kVp}$, produce una imagen más oscura (mayor contraste de tejidos) (Tabla II).

Los tres factores que tenemos que hay que tener en cuenta para mejorar el detalle de la imagen son disminuir la distancia del paciente al intensificador de imagen, utilizar colimación y disminuir el $\mathrm{kVp}$.

\section{EFECTOS BIOLÓGICOS Y DAÑINOS DE LA RADIACIÓN IONIZANTE}

Los rayos $\mathrm{X}$ actúan sobre los tejidos de dos maneras:

- Por un lado, cuando los rayos X interaccionan sobre un electrón de la capa externa del átomo del tejido que adsorbe la radiación parte de la energía es transferida al electrón en forma de energía cinética, causando que este salga del átomo. Entonces el rayo X es reflectado o dispersado de su dirección original pero con menor energía, produciendo una radiación dispersa. Esto se denomina "efecto Compton".

- Por otro lado, el efecto fotoeléctrico ocurre cuando un rayo $\mathrm{X}$ actúa sobre un electrón de una capa interna del átomo transfiriendo toda su energía, parte de la cual es usada para liberarse del átomo y salir. Entonces un electrón de una capa externa ocupa su lugar. Esto produce una emisión de radiación conocida como "radiación secundaria".

Ni la radiación dispersa producida por efecto Compton ni la radiación secundaria producida por el efecto fotoeléctrico contribuyen a la formación de la imagen, pero sí representan un tipo de radiación perjudicial que puede afectar a las personas cercanas al equipo de rayos $\mathrm{X}$ y es la que hay que tener en cuenta a la hora de la protección.

Los efectos dañinos de la radiación sobre el ser humano pueden ser de dos tipos:

- Los denominados “deterministas”, tales como la esterilidad, las cataratas, el eritema, los trastornos hematopoyéticos y el síndrome agudo por radiación, pueden evitarse del todo si las dosis que reciben las personas no sobrepasan determinados umbrales.

- Existen otros efectos, llamados "estocásticos" (la inducción del cáncer y algunos trastornos hereditarios), que no pueden evitarse por completo.

Se considera que cualquier exposición a la radiación ionizante, por pequeña que sea la dosis, puede contribuir a

TABLA II. FACTORES PARA MEJORAR EL DETALLE DE LA IMAGEN

1. Disminuir la distancia del paciente al intensificador de imagen

2. Utilizar colimación

3. Disminuir el kVp la inducción de cáncer, y si la exposición es de las gónadas puede ser causa de trastornos hereditarios. Esto se ha comprobado en animales, aunque hasta ahora no se ha descrito en las personas expuestas a radiaciones.

Los efectos estocásticos de las radiaciones ionizantes no se pueden evitar, pueden tardar años en manifestarse y su relación con la radiación puede incluso obviarse. Dosis y duración son aditivas y juntas determinan la exposición de radiación. Una mayor exposición implica más riesgo.

\section{DOSIS DE RADIACIÓN}

Como anteriormente se señalaba, no existe una cantidad de radiación que pueda considerarse segura para una persona.

La dosis máxima permisible es la dosis en el límite alto de radiación que se puede permitir que una persona reciba sin riesgo de efectos secundarios importantes. La dosis de radiación bajo este nivel posiblemente lleva una remota posibilidad de que aparezcan efectos secundarios clínicos. Por ejemplo, la dosis que se puede recibir al realizar un bloqueo epidural, por la exposición a la radiación dispersa, tomando las medidas necesarias de precaución, con una técnica y equipo adecuado y a $1 \mathrm{~m}$ de distancia, viene a ser de $0.03 \mathrm{mR}$. Aunque esto es relativamente pequeño, no debe conducirnos a una falsa sensación de seguridad porque, como sabemos, la dosis de radiación es acumulativa.

Frecuentemente, el médico que hace el bloqueo suele tener las manos cerca o encima del paciente. Esto puede producir dermatitis por radiación o efectos malignos a largo plazo. La exposición a la radiación de un arco de fluoroscopia sobre la piel varía entre 1 y $10 \mathrm{R} / \mathrm{min}$ (uso de rayos $\mathrm{X}$ en forma de pulsos), aunque puede subir hasta $40 \mathrm{R} / \mathrm{min}$ si utilizamos el modo continuo. Por ejemplo, la radiación por la exposición de la piel a una placa de tórax, columna lumbosacra o abdomen son unos $15 \mathrm{mR}, 250 \mathrm{mR}$ y 220 $\mathrm{mR}$, respectivamente. Esto quiere decir que 1 minuto de fluoroscopia con una entrada típica de $2 \mathrm{R} / \mathrm{min}$ equivale aproximadamente a unas 130 placas de tórax u 8 placas de abdomen. Las dosis que llegan a los órganos son menores que las que llegan a la piel, por la atenuación de los tejidos blandos.

La aparición de cataratas es otro factor que se debe considerar en caso de trabajar con rayos X. La dosis mínima requerida para producir una catarata progresiva es por encima de 200 rem (2 Sv) en una exposición única, aunque se puede producir con dosis superiores en exposiciones repetidas (por encima de 800 rem o $8 \mathrm{~Sv}$ ). El periodo de latencia entre la radiación y la aparición de cataratas está relacionado con la dosis, aunque necesita una media de 8 años para desarrollarse (2).

Mientras que la exposición en una Unidad de Dolor con arco de fluoroscopia portátil es posiblemente menor que en 
una Unidad de Hemodinámica (coronariografía, angiografía, estudios electrofisiológicos), para el personal a más de $1 \mathrm{~m}$ de distancia, si se utiliza en exceso, puede ser similar (cientos de $\mathrm{mR} / \mathrm{h}$ ).

La radiación diseminada debe ser también importante para el resto de personas que están alrededor de la fuente de rayos X. La diseminación aumenta si el paciente es obeso o grande, pues normalmente necesita mayor dosis para producir una imagen adecuada.

Se considera que una dosis total mayor de $1 \mathrm{~Sv}$ (1.000 rem) puede producir efectos significativos como náuseas, cansancio, alteración hematológica, alteración intestinal, alopecia y dermatitis (3).

\section{LÍMITES DE DOSIS EN LA REGLAMENTACIÓN ESPAÑOLA}

Se ha estimado que una persona que no está expuesta profesionalmente a la radiación puede recibir alrededor de 3,6 mSv/año o 360 mrem/año, de los que una pequeña cantidad, un $15 \%$ aproximadamente, puede corresponder a radiación recibida por exposición a procedimientos médicos comunes como radiografías (4). Sin embargo, esta cantidad puede incrementarse significativamente si se exponen a otros procedimientos médicos menos frecuentes, como la tomografía axial computarizada (TC).

Se han establecido unos límites anuales de dosis recibidas en el Reglamento de Protección Sanitaria contra radiaciones ionizantes, no teniendo en cuenta las dosis debidas al fondo radiactivo natural (5) (Tabla III).

\section{MONITORIZACIÓN DE LA RADIACIÓN}

Es necesario medir de forma numérica la exposición a la radiación ionizante para evitar los efectos nocivos sobre las personas expuestas. Para ello se utilizan los dosímetros. Estos aparatos producen una señal en forma de densidad óptica o acumulación de energía al ser expuestos a la radiación y, por lo tanto, indican una estimación de la acumulación de exposición sobre un periodo de tiempo (se recomienda leer el dosímetro de forma mensual) en el individuo que lleva el aparato.

En caso de profesionales con mayor tiempo de exposición, como ocurre en aquellos que trabajan en la sala de hemodinámica o los que realizamos técnicas intervencionistas, se recomienda llevar el dosímetro por dentro de los mandiles en la zona pectoral, además de uno externo en muñeca o en dedo en forma de anillo, donde se recibe el haz de radiación directamente. Si se utiliza fluoroscopia de forma esporádica un dosímetro en el bolsillo suele ser suficiente.

\section{Tipos de dosímetros}

Los métodos más usuales para llevar a cabo una dosimetría personal son:

- Dosímetro de cámara de ionización: posee una apariencia externa de bolígrafo. Tiene la ventaja de que se puede obtener la lectura de la exposición inmediatamente después de recibirla. En cambio, no es de registro permanente. Su costo es más alto que el de las películas fotográficas, pero se pueden usar repetidas veces. Es sensible a golpes y otros maltratos (Fig. 3).

- Dosímetro de película: está basado en los mismos principios que las películas fotográficas impresionadas por acción de la luz o que la de las radiografías con rayos X. Mediante un proceso químico, denominado revelado, se puede poner de manifiesto la imagen latente dejada por la radiación en la película dosimétrica. Es el utilizado más comúnmente (Fig. 4).

- Dosímetro de termoluminiscencia: contiene sustancias cristalinas como fluoruro de litio $(\mathrm{LiF})$ o fluoruro de calcio $\left(\mathrm{CaF}_{2}\right)$, que suelen retener los electrones emitidos por la radiación. Cuando posteriormente se calientan estos cristales, los electrones atrapados vuelven a caer a sus estados originales emitiendo luz al mismo tiempo (de allí el nombre de termoluminis-

TABLA III. LÍMITES ANUALES DE DOSIS SEGÚN EL REGLAMENTO DE PROTECCIÓN SANITARIA CONTRA RADIACIONES IONIZANTES, SEGÚN EL R.D. 783/2001

\begin{tabular}{|c|c|c|c|}
\hline \multirow{2}{*}{ Personas } & \multirow{2}{*}{ Dosis efectiva } & \multicolumn{2}{|c|}{ Dosis equivalente } \\
\hline & & Cristalino & Piel/extremidades \\
\hline Trabajador externo & $50 \mathrm{mSv} / \mathrm{año}$ & $150 \mathrm{mSv} / \mathrm{año}$ & 500 mSv/año \\
\hline Personal en formación & $6 \mathrm{mSv} / \mathrm{año}$ & $50 \mathrm{mSv} / \mathrm{año}$ & $150 \mathrm{mSv} / \mathrm{año}$ \\
\hline Embarazadas & \multicolumn{3}{|c|}{$1 \mathrm{mSv}$ durante el embarazo* } \\
\hline Público y trabajadores no expuestos & $1 \mathrm{mSv} / \mathrm{año}$ & 15 mSv/año & $50 \mathrm{mSv} / \mathrm{año}$ \\
\hline
\end{tabular}

*A partir de la declaración del embarazo, tomar las medidas de protección necesarias para no superar $1 \mathrm{mSv}$ en el feto. 


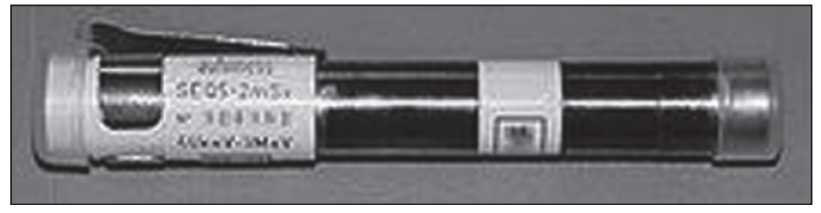

Fig. 3. Dosímetro de cámara de ionización.

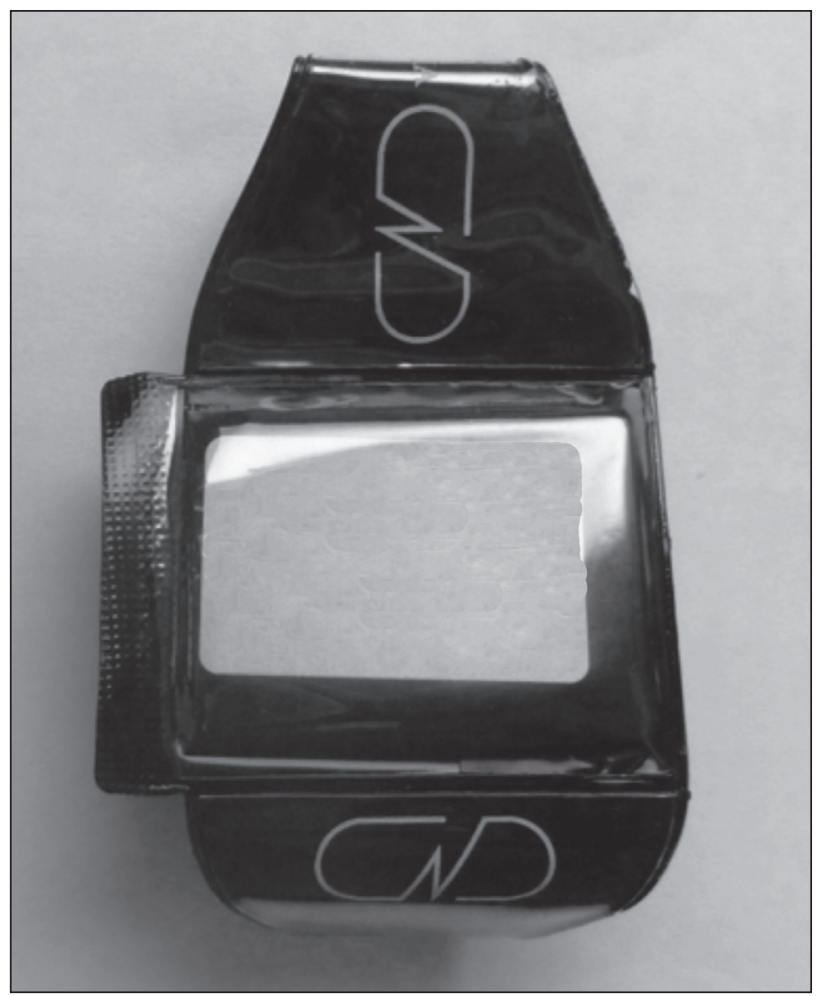

Fig. 4. Dosímetro de película.

cencia). La cantidad de luz emitida se mide con un fotomultiplicador. Es de costo moderado, resistente y puede ser usados varias veces. Es más preciso que los de película, pero se requiere un equipo especial para efectuar las lecturas (Fig. 5).

\section{CÓMO REDUCIR LA EXPOSICIÓN A LA RADIACIÓN}

La dosis recibida por un individuo que esté cerca de una fuente de radiación está determinada por tres factores:

1. La distancia entre la fuente y el individuo.

2. El tiempo de radiación.

3. La materia interpuesta entre una y otro.

1. Los rayos $X$ de una fuente radiactiva se propagan por el aire, siguiendo la ley de proporcionalidad inversa

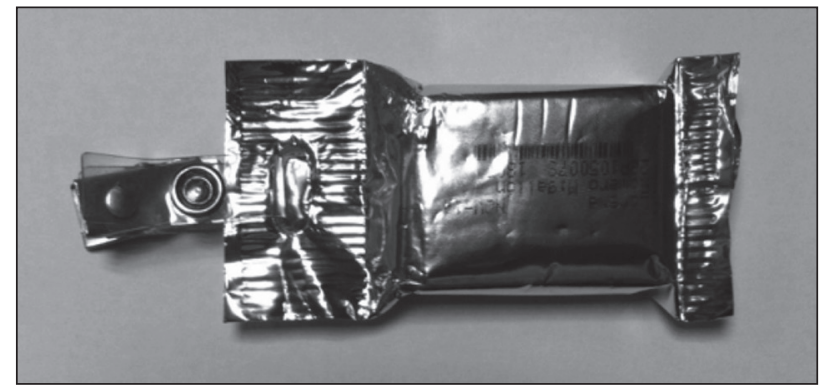

Fig. 5. Dosímetro de termoluminiscencia.

al cuadrado de la distancia, de modo que al alejarse de la fuente, doblando la distancia del paciente, la exposición sería una cuarta parte. De la misma forma, al acercarse a la fuente la intensidad de radiación aumenta en la misma proporción.

La mayor fuente de radiación es el paciente, que sirve como medio de dispersión de radiación. Generalmente, la dispersión de la radiación de un paciente a 1 m es aproximadamente el $0,1 \%$ de la radiación que entra.

Es importante conocer que cuando empleamos la visión lateral con el arco la dispersión de radiación en el lado de entrada de rayos X es 2-3 veces mayor que en el lado opuesto del intensificador de imagen. Por lo tanto, estando en el lado del intensificador de imagen se ofrece menor exposición.

2. Minimizar el tiempo de exposición, usando el menor tiempo posible de fluoroscopia e imagen continua y empleando cuanto más sea posible la imagen congelada o en pulsos.

La mayoría de equipos tienen un límite automático de 5 minutos, en forma de pitido o parada de la exposición. En algunos aparatos antiguos este aviso no existe y por ello es necesario mantener la cuenta del tiempo de radiación utilizado.

3. Es importante también interponer entre la fuente de radiación y el operador algún material absorbente de la radiación. Toda radiación, al atravesar la materia, sufre una disminución o atenuación de su intensidad. Según sea el tipo y la energía de la radiación y la atenuación que se quiera conseguir habrá que utilizar distintos tipos y espesores de blindajes. Una hoja de papel es suficiente para detener la radiación alfa; la radiación beta sería totalmente absorbida por unos pocos centímetros de algún material ligero como madera, vidrio, plástico. Para construir un blindaje adecuado para los rayos $\mathrm{X}$ o la radiación gamma es preciso emplear materiales más pesados (hormigón, plomo, etc.). 


\section{Protectores de plomo}

Los protectores de plomo adecuados son un requerimiento obligado en la sala de fluoroscopia y existen varias opciones: mandiles, guantes, protector de tiroides y gafas.

Los mandiles de plomo absorben el 90-95\% de la radiación dispersa que llega al tronco. Si la corriente de los rayos $\mathrm{X}$ es $100 \mathrm{kVp}$ un mandil de plomo debe tener al menos $0,25 \mathrm{~mm}$ de espesor. Un mayor espesor, de 0,5 a $1 \mathrm{~mm}$, es obviamente mejor, aunque a expensas de mayor peso del equipo, lo que puede ser un impedimento importante si se lleva durante mucho tiempo. Los mandiles que rodean por delante y detrás son recomendables cuando el clínico tiene que volverse frecuentemente. Las piernas también deberían ser protegidas.

El protector de tiroides es importante para disminuir el riesgo de cáncer de tiroides. Este debe tener al menos 0,5 $\mathrm{mm}$ de espesor.

La utilización de guantes plomados está recomendada, con una capa de al menos $0,25 \mathrm{~mm}$, cuando las manos del especialista están en la dirección del rayo. Incluso con ello, las manos deberían estar fuera del campo. Llevar un dosímetro de anillo es una manera eficaz de evaluar la radiación recibida en ellas.

Hay que usar gafas con cristales transparentes pero que contienen una protección plomada equivalente a $0,35 \mathrm{~mm}$, ya que pueden atenuar de forma significativa la radiación diseminada que afecta a las lentes oculares y reducir la acumulación de radiación. Se recomiendan para aquellas personas que reciban una radiación mensual en el dosímetro por encima de $4 \mathrm{mSv}$ ( $400 \mathrm{mrem}$ ). Además, se recomienda colocar en las gafas unos cristales protectores laterales plomados que contengan un equivalente a $0,5 \mathrm{~mm}$.

Los protectores de la cabeza suelen ser en forma de placas planas, transparentes, normalmente suspendidas del techo y que separan al médico del paciente.

Recomendaciones para el personal expuesto cuando se utiliza la fluoroscopia:

- Solo los profesionales necesarios deben estar en el área de radiación.

- No se debe coger al paciente durante la radiación.

- Todos deben llevar protección apropiada.

- Al comenzar a utilizar los rayos X se debe avisar al personar de ello.

Además, los principios de protección radiológica recomiendan:

- Colocar la fuente de rayos X separada al menos a 30 $\mathrm{cm}$ del paciente.

- Colocar el intensificador de imagen lo más cerca posible del paciente.

- Usar el colimador lo más cerrado posible.

- Usar magnificación electrónica e imágenes fijas lo más frecuentemente posible.
- Utilizar el arco de fluoroscopia en habitaciones que estén equipadas con medidas de protección adecuadas (generalmente con plomo o cemento) en caso de usarlo frecuentemente.

\section{CONSENTIMIENTO INFORMADO PARA LA EXPOSICIÓN A RADIACIÓN}

La información del uso de fluoroscopia en los procedimientos de tratamiento del dolor suele ser omitida al explicar a los pacientes qué les vamos a hacer.

Es recomendable hablar de los riesgos, beneficios y alternativas cuando se usan los rayos $\mathrm{X}$, así como preguntar a las mujeres por la posibilidad de embarazo y explicar el posible daño al feto.

Deberíamos notificar que la cantidad de radiación recibida suele ser mínima, aunque no es posible decir con exactitud la cantidad de radiación que se puede recibir: aproximadamente está entre la recibida por una placa de tórax y lo recibido por un procedimiento en hemodinámica. Además, se puede explicar que la radiación es menor cuando la exposición es solo en una parte del cuerpo que cuando se hace de forma más generalizada.

Los principales riesgos a largo plazo son la posibilidad de cáncer y de alteraciones cromosómicas.

Los posibles efectos a corto plazo son lesiones en la piel, como eritema, o incluso quemaduras de segundo grado.

CORRESPONDENCIA:

José M. ${ }^{a}$ Hernández García

Departamento de Anestesia y Tratamiento del Dolor

Hospital Universitario Madrid Montepríncipe

Avda. de Montepríncipe, 25

28660 Boadilla del Monte, Madrid

e-mail: jmhernandez@madridsindolor.com

\section{BIBLIOGRAFÍA}

1. Hernández García JM, Vidal Marcos A, Gasco García C. Encuesta acerca de la utilización de fluoroscopia en el tratamiento del dolor. ¿Lo hacemos correctamente? Rev Esp Anestesiol Reanim 2012;59(8):430-5.

2. Fishman SM, Smith H, Meleger A, Seibert JA. Radiation Safety in Pain Medicine. Reg Anes Pain Med 2002;3(27):296-305.

3. Hall EJ. Radiobiology for the Radiologist; 4a ed. Philadelphia, PA: Lippincott. 1994.

4. Statkiewicz-Sherer MA, Viscanti PJ, Ritenour ER. Radiation Protection in Medical Radiography. 3rd ed. St. Louis, MO: Mosby. 1998.

5. Reglamento de Protección Sanitaria contra Radiaciones Ionizantes (Real Decreto 783/2001, de 6 de julio de 2001. B.O.E. n. ${ }^{\circ} 178,26$ de julio de 2001). 